\title{
FIXED-POINT AND COINCIDENCE THEOREMS \\ FOR SET-VALUED MAPS WITH NONCONVEX \\ OR NONCOMPACT DOMAINS \\ IN TOPOLOGICAL VECTOR SPACES
}

\author{
KAZIMIERZ WŁODARCZYK AND DOROTA KLIM
}

Received 9 January 2002

\begin{abstract}
A technique, based on the investigations of the images of maps, for obtaining fixed-point and coincidence results in a new class of maps and domains is described. In particular, we show that the problem concerning the existence of fixed points of expansive set-valued maps and inner set-valued maps on not necessarily convex or compact sets in Hausdorff topological vector spaces has a solution. As a consequence, we prove a new intersection theorem concerning not necessarily convex or compact sets and its applications. We also give new coincidence and section theorems for maps defined on not necessarily convex sets in Hausdorff topological vector spaces. Examples and counterexamples show a fundamental difference between our results and the well-known ones.
\end{abstract}

\section{Introduction}

Suppose that $E$ is a Hausdorff topological vector space, $C \subset E, C \neq \varnothing$. In fixedpoint and coincidence theory and its applications, a great part of the vast literature in the last century concerns conditions on $C, E, F$, and $G$ guaranteeing the existence of fixed points or coincidences of set-valued maps $F: C \rightarrow 2^{E}$ and $G: C \rightarrow 2^{E}$. In various methods of investigations, the assumptions that the maps are inner and $C$ are convex compact subsets of $E$ play the crucial role (see, e.g., $[5,6,7,15,16,17,18,19,20,21,22,23,24,25,27,30,33,41])$. The general topic of fixed points and coincidences for set-valued maps on convex compact sets, originating mainly with the work of Kakutani [30], Bohnenblust and Karlin [5], Glicksberg [23], Fan [15, 16, 17, 18, 19, 20, 21, 22], and Browder [6, 7], has been well developed in various directions.

In the past decade, there was a renewed interest in the fixed-point and coincidence theory of set-valued maps in topological vector spaces (see, e.g., [2, $3,8,9,10,11,12,28,36,37,38,39,40,42,43,44,45,46,47,48]$ ), partially due to new and powerful methods of investigations introduced into it (notably

Copyright (C) 2003 Hindawi Publishing Corporation Abstract and Applied Analysis 2003:1 (2003) 1-18 2000 Mathematics Subject Classification: 47H10, 47H04

URL: http://dx.doi.org/10.1155/S1085337503207028 
based on those introduced by Fan and Browder). Most of the work has centered around the fixed-point and coincidence theory of maps on convex compact sets, but there are also a considerable number of papers devoted to maps on nonconvex and noncompact sets (see, e.g., $[8,45]$ ).

There exist a number of introductions to and surveys of fixed-point and coincidence theory. We mention [47] among the more recent ones but also some elder ones $[14,49,50]$. See also many references therein.

A natural question arises: whether expansive set-valued maps and inner setvalued maps on not necessarily convex or compact sets have fixed points and, as a consequence, theorems of intersection type hold and whether the maps $F: C \rightarrow 2^{E}$ and $G: C \rightarrow 2^{E}$ on not necessarily convex sets in Hausdorff topological vector spaces have coincidences. The affirmative answers are given in this paper. Using a technique based on the investigation of the images of maps, we obtain a number of new fixed-point, coincidence, intersection, and section theorems of Fan-Browder type. Examples and counterexamples show a fundamental difference between our results and the known results of the above-mentioned authors.

\section{Fixed points and coincidences of expansive set-valued maps on not necessarily convex or compact sets in topological vector spaces}

Let $C$ be a subset of a Hausdorff topological vector space $E$ over $\mathbb{K}(\mathbb{K}=\mathbb{R}$ or $\mathbb{C}$ ). A set-valued map $F: C \rightarrow E$ (which will always be denoted by capital letters) is a map which assigns a unique $F(c) \in 2^{E}$ (here $2^{E}$ denotes the family of all subsets of $E$ ) to each $c \in C$. We say that $c \in C$ is a fixed point of $F: C \rightarrow 2^{E}$ if $c \in F(c)$. We say that a map $F: C \rightarrow 2^{E}$ is expansive if $C \subset F(C)$ where $F(C)=\bigcup_{c \in C} F(c)$.

Maps in the usual sense will be considered as special (single-valued) setvalued maps and these ordinary maps will always be denoted by small letters $f: C \rightarrow E$.

We prove the following theorem.

Theorem 2.1. Let $C$ be a nonempty subset of a Hausdorff topological vector space $E$ over $\mathbb{R}$, let $F: C \rightarrow 2^{E}$, and let $K$ be a convex subset of $E$. Assume that the following conditions hold:

(i) $C \subset K \subset F(C)$;

(ii) $F(C)$ is a compact subset of $E$;

(iii) for each $c \in C, F(c)$ is open in $F(C)$;

(iv) for each $y \in K, F^{-1}(y)=\{c \in C: y \in F(c)\}$ is nonempty and convex.

Then there exists $u \in C$ such that $u \in F(u)$.

Proof. By (iii), the compact set $F(C)$ is covered by the sets $F(c), c \in C$, which are open in $F(C)$. Clearly, there exists a finite set $\left\{c_{1}, \ldots, c_{n}\right\} \subset C$ such that $F\left(c_{i}\right)$ are nonempty, $1 \leq i \leq n$, and $F(C)=\bigcup_{i=1}^{n} F\left(c_{i}\right)$. Let $\left\{\varphi_{1}, \ldots, \varphi_{n}\right\}$ be a partition of 
unity with respect to this cover, that is, a finite family of real-valued nonnegative continuous maps $\varphi_{i}$ on $F(C)$ such that $\varphi_{i}$ vanish outside $F\left(c_{i}\right)$ and are less than or equal to one everywhere, $1 \leq i \leq n$, and $\sum_{i=1}^{n} \varphi_{i}(y)=1$ for all $y \in F(C)$.

Let $\sigma$ be a simplex spanned by points $c_{1}, \ldots, c_{n}$ and let $\varphi: F(C) \rightarrow \sigma$ be a continuous map defined by the formula $\varphi(y)=\sum_{i=1}^{n} \varphi_{i}(y) c_{i}, y \in F(C)$. Clearly, $\sigma \subset K \subset F(C)$ and hence $\varphi(\sigma) \subset \varphi(K) \subset \varphi(F(C)) \subset \sigma$.

If $y \in K$ is arbitrary and fixed and $\varphi_{i}(y) \neq 0$ for some $i \in\{1, \ldots, n\}$, then $y \in F\left(c_{i}\right)$, so $c_{i} \in F^{-1}(y)$. As a consequence, for each $y \in K, \varphi(y)$ is a convex linear combination of points of $F^{-1}(y)$ and by (iv), we get for each $y \in K$,

$$
\varphi(y) \in F^{-1}(y), \quad \varphi(y) \in C \text {. }
$$

From Brouwer's theorem, we get $u=\varphi(u)$ for some $u \in \sigma$ and hence, since $\sigma \subset K$, by (2.1), $u=\varphi(u) \in F^{-1}(u) \subset C$, and therefore, $u \in F(u)$ and $u \in C$, as required.

By using various sets $K$, a number of variations of Theorem 2.1 can be obtained, of which the following two are typical.

Theorem 2.2. Let $C$ be a nonempty subset of a Hausdorff topological vector space E over $\mathbb{R}$ and let $F: C \rightarrow 2^{E}$. Assume that the following conditions hold:

(i) $F$ is expansive, that is, $C \subset F(C)$;

(ii) $F(C)$ is convex;

(iii) $F(C)$ is a compact subset of $E$;

(iv) for each $c \in C, F(c)$ is open in $F(C)$;

(v) for each $y \in F(C), F^{-1}(y)=\{c \in C: y \in F(c)\}$ is nonempty and convex.

Then there exists $u \in C$ such that $u \in F(u)$.

Proof. We use Theorem 2.1 for $K=F(C)$.

THEOREM 2.3. Let $C$ be a nonempty subset of a Hausdorff topological vector space $E$ over $\mathbb{R}$ and let $F: C \rightarrow 2^{E}$. Assume that the following conditions hold:

(i) $F$ is expansive, that is, $C \subset F(C)$;

(ii') $C$ is convex;

(iii) $F(C)$ is a compact subset of $E$;

(iv) for each $c \in C, F(c)$ is open in $F(C)$;

$\left(\mathrm{v}^{\prime}\right)$ for each $y \in C, F^{-1}(y)=\{c \in C: y \in F(c)\}$ is nonempty and convex.

Then there exists $u \in C$ such that $u \in F(u)$.

Proof. Indeed, if $\varphi$ and $\sigma$ are as in the proof of Theorem 2.1 and $C$ is convex, then $\sigma \subset C \subset F(C)$, and we may use Theorem 2.1 for $K=C$.

Example 2.4. (a) Let $E=\mathbb{R}^{2}$, let $T$ be a closed triangle with vertices $(0,0),(1,0)$ and $(0,1)$, and let $C=C_{1} \cup\{(0,0)\} \cup C_{2}$ where $C_{1}=\left\{c=\left(c_{1}, 0\right): 0<c_{1} \leq 1\right\}$ and $C_{2}=\left\{c=\left(0, c_{2}\right): 0<c_{2} \leq 1\right\}$. Define sets $P=\left\{c=\left(c_{1}, c_{2}\right):\left|c_{2}-c_{1}\right|<1 / 2\right\}$, $H_{1}=\left\{c=\left(c_{1}, c_{2}\right): c_{2}<c_{1}\right\}$ and $H_{2}=\left\{c=\left(c_{1}, c_{2}\right): c_{2}>c_{1}\right\}$. If $F(C)=T$ where 
4 Fixed-point and coincidence theorems

$F(0,0)=T \cap P, F(c)=T \cap H_{1}$ for $c \in C_{1}$, and $F(c)=T \cap H_{2}$ for $c \in C_{2}$, then the assumptions of Theorem 2.2 are satisfied, $C$ is nonconvex and $\operatorname{Fix}(F)=C$.

(b) Let $E=\mathbb{R}, C=(1 ; 3), F(C)=[0 ; 4]$ where $F(c)=[0 ; 2)$ for $c \in(1 ; 2), F(c)=$ $(2 ; 4]$ for $c \in(2,3)$ and $F(2)=(1 ; 3)$. Then the assumptions of Theorem 2.2 are satisfied, $C$ is noncompact and $\operatorname{Fix}(F)=C$.

If in Theorems 2.2 or 2.3 we omit at least one of the assumptions, then we can construct a counterexample.

Example 2.5. (a) The condition on $F(C)$ in (i) cannot be omitted as the following two counterexamples show:

$\left(\mathrm{A}_{1}\right)$ if $E=\mathbb{R}, C=(1 ; 4), F(C)=[2 ; 5]$ where $F(c)=[2 ; 5]$ for $c \in(1 ; 2)$ and $F(c)=(4 ; 5]$ for $c \in[2 ; 4)$, then assumptions (ii), (iii), (iv), and (v) are satisfied, $C \not \subset F(C), F(C) \not \subset C$, and $\operatorname{Fix}(F)=\varnothing$;

$\left(\mathrm{A}_{2}\right)$ if $E=\mathbb{R}^{2}, C=\left\{c=\left(c_{1}, c_{2}\right):\left|c_{1}\right| \leq 1,\left|c_{2}\right| \leq 2\right\}, C=C_{1} \cup C_{2} \cup C_{3} \cup$ $\left(-C_{3}\right) \cup C_{4}$ where $C_{1}=\{(0,-2)\}, C_{2}=\{(0,2)\}, C_{3}=\left\{c=\left(c_{1}, c_{2}\right):-1\right.$ $\left.\leq c_{1}<0,\left|c_{2}\right| \leq 2\right\}$, and $C_{4}=\left\{c=\left(c_{1}, c_{2}\right): c_{1}=0,\left|c_{2}\right|<2\right\}, F\left(C_{1}\right)=D_{1}$, $F\left(C_{2}\right)=D_{2}, F\left(C_{3}\right)=D_{3}, F\left(-C_{3}\right)=-D_{3}, F\left(C_{4}\right)=D_{3} \cup\left(-D_{3}\right)$, and if $F(C)=\left\{y=\left(y_{1}, y_{2}\right):\left|y_{1}\right| \leq 1,\left|y_{2}\right| \leq 1\right\}$ where $D_{1}=\left\{y=\left(y_{1}, y_{2}\right):\left|y_{1}\right|<\right.$ $\left.1 / 2,\left|y_{2}\right| \leq 1\right\}, D_{2}=\left\{y=\left(y_{1}, y_{2}\right): 1 / 2<\left|y_{1}\right| \leq 1,\left|y_{2}\right| \leq 1\right\}$, and $D_{3}=$ $\left\{y=\left(y_{1}, y_{2}\right): 0<y_{1} \leq 1,\left|y_{2}\right| \leq 1\right\}$, then assumptions (ii), (iii), (iv), and (v) are satisfied, $F(C) \subset C, F(C) \neq C$, and $\operatorname{Fix}(F)=\varnothing$.

(b) The assumption that $F(C)$ is convex or $C$ is convex is necessary. Indeed, let $E=\mathbb{R}^{2}, C=C_{0} \cup\left(-C_{0}\right)$ where $C_{0}=\left\{c=\left(c_{1}, 0\right): c_{1} \in(1 ; 2)\right\}, F(c)=D_{0}$ for $c \in$ $-C_{0}$, and $F(c)=-D_{0}$ for $c \in C_{0}$ where $D_{0}=\left\{y=\left(y_{1}, y_{2}\right):\left\|\left(y_{1}, y_{2}\right)-(3 / 2,0)\right\| \leq\right.$ 1\}. Then assumptions (i), (iii), (iv), and (v) are satisfied, while (ii) and (ii') are not, and $\operatorname{Fix}(F)=\varnothing$.

(c) The assumption that $F(C)$ is compact is necessary. Indeed, let $E=\mathbb{R}$, $C=(0 ; 1), F(c)=(0 ; c)$ for $1 / 2 \leq c<1, F(c)=(1 / 2+c ; 1)$ for $0<c<1 / 2$. Then assumptions (i), (ii), (iv), and (v) are satisfied and $\operatorname{Fix}(F)=\varnothing$.

(d) Assumption (iv) is necessary. Indeed, let $E=\mathbb{R}, C=(1 ; 4), F(C)=[1 ; 4]$ where $F(c)=[1 ; 2) \cup(3 ; 4]$ for $c \in(2 ; 3), F(c)=(2 ; 5 / 2)$ for $c \in(1 ; 2), F(c)=$ $[5 / 2 ; 3)$ for $c \in(3 ; 4), F(2)=\{3\}$ and $F(3)=\{2\}$. Thus assumptions (i), (ii), (iii), and $(\mathrm{v})$ are satisfied and $\operatorname{Fix}(F)=\varnothing$.

(e) Assumption (v) (or $\left.\left(\mathrm{v}^{\prime}\right)\right)$ is necessary. Indeed, let $E=\mathbb{R}, C=(1 ; 5), F(C)=$ $[1 ; 5]$ where $F(c)=(2 ; 3) \cup(4 ; 5]$ for $c \in(1 ; 2), F(c)=[1 ; 2) \cup(3 ; 4)$ for $c \in(2 ; 3)$, $F(c)=[1 ; 3) \cup(4 ; 5]$ for $c \in(3 ; 4), F(c)=[1 ; 2) \cup(3 ; 4]$ for $c \in(4 ; 5), F(2)=$ $(2 ; 5], F(3)=[1 ; 3) \cup(3 ; 5]$, and $F(4)=[1 ; 4)$. Then $F^{-1}(4)=\{2,3\}$ is nonconvex. Thus assumptions (i), (ii'), (iii), and (iv) are satisfied, while $\left(\mathrm{v}^{\prime}\right)$ is not, and $\operatorname{Fix}(F)=\varnothing$.

We say that a single-valued map $f: C \rightarrow E$ and a set-valued map $F: C \rightarrow 2^{E}$ have a coincidence if $f(c) \in F(c)$ for some $c \in C$. 
The following theorem is a generalization of the above one.

Theorem 2.6. Let $C$ be a nonempty convex subset of a Hausdorff topological vector space $E$ over $\mathbb{R}$, let $F: C \rightarrow 2^{E}$ be an expansive map, and let $f: C \rightarrow E$ be a single-valued continuous map such that $f(C) \subset F(C)$. Assume that the following conditions hold:

(i) $F(C)$ is a compact subset of $E$;

(ii) for each $c \in C, F(c)$ is open in $F(C)$;

(iii) for each $y \in f(C), F^{-1}(y)=\{c \in C: y \in F(c)\}$ is nonempty and convex.

Then there exists $u \in C$ such that $f(u) \in F(u)$.

Proof. Let $\varphi$ and $\sigma$ be as in the proof of Theorem 2.1. We have $\varphi: F(C) \rightarrow \sigma$, $\sigma \subset C$, and $y \in F(\varphi(y))$ for each $y \in f(C)$. On the other hand, $\varphi \circ f: \sigma \rightarrow \sigma$ and, by the theorem of Brouwer, $(\varphi \circ f)(u)=u$ for some $u \in \sigma$. Consequently, $f(u) \in F(\varphi(f(u)))=F(u)$.

\section{Fixed points and coincidences of set-valued inner maps on not necessarily convex sets in topological vector spaces}

We say that a map $F: C \rightarrow 2^{E}$ is inner if $F(C) \subset C$. This section is devoted to new fixed-point and coincidence theorems for set-valued inner maps on not necessarily convex sets.

We have the following theorem.

TheOREM 3.1. Let $C$ be a nonempty compact subset of a Hausdorff topological vector space $E$ over $\mathbb{R}$ and let $F: C \rightarrow 2^{E}$ be an inner map such that $F(C)$ is a convex subset of E. Assume that the following conditions hold:

(i) for each $c \in C, F(c)$ is nonempty and convex;

(ii) for each $y \in F(C), F^{-1}(y)=\{c \in C: y \in F(c)\}$ is open in $C$.

Then there exists $u \in C$ such that $u \in F(u)$.

Proof. In virtue of (i) and (ii), there exists a finite set $\left\{y_{1}, \ldots, y_{n}\right\} \subset F(C)$ such that $F^{-1}\left(y_{i}\right)$ are nonempty, $1 \leq i \leq n$, and $C=\bigcup_{i=1}^{n} F^{-1}\left(y_{i}\right)$. Let $\left\{\varphi_{1}, \ldots, \varphi_{n}\right\}$ be a partition of unity with respect to this cover, let $\sigma$ be a simplex spanned by points $y_{1}, \ldots, y_{n}$, and let a continuous map $\varphi: C \rightarrow \sigma$ be defined by the formula $\varphi(c)=\sum_{i=1}^{n} \varphi_{i}(c) y_{i}, c \in C$. Note that $\sigma \subset F(C) \subset C$ and, consequently, $\varphi(\sigma) \subset \sigma$.

If $c \in C$ and $\varphi_{i}(c) \neq 0$ for some $i \in\{1, \ldots, n\}$, then $c \in F^{-1}\left(y_{i}\right)$, thus $y_{i} \in F(c)$. By (i), for each $c \in C$,

$$
\varphi(c) \in F(c) .
$$

On the other hand, from Brouwer's theorem, we get that $u=\varphi(u)$ for some $u \in \sigma$ and, by (3.1), we have $u=\varphi(u) \in F(u)$, as required. 
6 Fixed-point and coincidence theorems

Recall that a map $F: C \rightarrow 2^{E}$ is called upper semicontinuous if, for each $c \in C$ and any open set $V$ containing $F(c)$, there is an open set $U$ containing $c$ such that $F(U \cap C) \subset V$ (for details, see [4]). A map $F: C \rightarrow 2^{E}$ is called upper demicontinuous on $C$ (after Fan [20]) if, for each $c \in C$ and any open half-space $H$ in $E$ containing $F(c)$, there is a neighbourhood $N(c)$ of $c$ in $C$ such that $F(x) \subset H$ for each $x \in N(c)$. It is clear that the condition of upper semicontinuity is stronger than that of upper demicontinuity.

Let $C$ and $D$ be nonempty sets. The maps $F: C \rightarrow 2^{D}$ and $G: D \rightarrow 2^{C}$ are said to have a coincidence if there exists $(u, v) \in C \times D$ such that $v \in F(u)$ and $u \in G(v)$.

We now establish the following theorem.

THEOREM 3.2. Let $C$ be a nonempty compact subset of a Hausdorff locally convex topological vector space $E$ over $\mathbb{R}$, let $F: C \rightarrow 2^{E}$ be an inner map, and let $G: C \rightarrow 2^{E}$ be an upper demicontinuous map such that $G(C) \subset F(C)$. Assume that the following conditions hold:

(i) $F(C)$ is a closed convex subset of $E$;

(ii) for each $c \in C, F(c)$ is nonempty and convex;

(iii) for each $y \in F(C), F^{-1}(y)=\{c \in C: y \in F(c)\}$ is open in $C$;

(iv) for each $c \in C, G(c)$ is a nonempty closed convex subset of $F(C)$.

Then there exists $(u, v) \in C \times F(C)$ such that $u \in F(v)$ and $v \in G(u)$.

Proof. Let $\varphi$ and $\sigma$ be as in the proof of Theorem 3.1. Since $\sigma \subset F(C) \subset C$ and $\varphi: C \rightarrow \sigma$, then $G \circ \varphi: F(C) \rightarrow 2^{F(C)}$ and $G \circ \varphi$ is upper demicontinuous on the compact convex set $F(C)$. By [20, Theorem 6], there exists $v \in F(C)$ such that $v \in G(\varphi(v))$. Moreover, in virtue of (3.1), $\varphi(v) \in F(v)$. This implies the assertion for $u=\varphi(v)$.

\section{Intersection theorem with applications on not necessarily convex or compact sets in topological vector spaces}

Various intersection theorems concerning convex and compact sets, with their applications, are given in $[6,17,18,21,22,33,35]$. From Theorem 2.2, we get the following new intersection theorem.

Theorem 4.1. Let $E$ be a Hausdorff topological vector space over $\mathbb{R}$ and let $n \geq 2$. Let $C_{1}, \ldots, C_{n}$ be nonempty (not necessarily convex or compact) subsets of $E$, let $K_{1}, \ldots, K_{n}$ be compact and convex subsets of $E$, let $S_{1}, \ldots, S_{n}$ be nonempty subsets of $E^{n}$, and let $C=\prod_{j=1}^{n} C_{j}, K=\prod_{j=1}^{n} K_{j}$, and $S=\bigcup_{j=1}^{n} S_{j}$. Assume that the following properties hold:

(i) $C \subset K=S$;

(ii) for each $i, 1 \leq i \leq n$, and for each point $\left(y_{1}, \ldots, y_{i-1}, y_{i+1}, \ldots, y_{n}\right)$ of $\prod_{j \neq i}^{n} K_{j}$, the section $S_{i}\left(y_{1}, \ldots, y_{i-1}, y_{i+1}, \ldots, y_{n}\right)$, formed by all points $c_{i} \in C_{i}$ such that $\left(y_{1}, \ldots, y_{i-1}, c_{i}, y_{i+1}, \ldots, y_{n}\right) \in S_{i}$, is a nonempty convex subset of $C_{i}$; 
(iii) for each $i, 0 \leq i \leq n$, and for each point $c_{i} \in C_{i}$, the section $S_{i}\left(c_{i}\right)$, formed by all points $\left(y_{1}, \ldots, y_{i-1}, y_{i+1}, \ldots, y_{n}\right)$ of $\prod_{j \neq i}^{n} K_{j}$ such that

$$
\left(y_{1}, \ldots, y_{i-1}, c_{i}, y_{i+1}, \ldots, y_{n}\right) \in S_{i}
$$

is an open subset of $\prod_{j \neq i}^{n} K_{j}$.

Then $C \cap \bigcap_{i=1}^{n} S_{i} \neq \varnothing$.

Proof. Define $F: C \rightarrow 2^{K}$ as follows. Fix a point $c \in C$ and let $y \in K$. We say that $y \in F(c)$ if and only if, for each $i \in\{1, \ldots, n\},\left(y_{1}, \ldots, y_{i-1}, c_{i}, y_{i+1}, \ldots, y_{n}\right) \in S_{i}$.

Write $c \in C$ in the form $c=\left(c_{1}, \ldots, c_{i-1}, c_{i}, c_{i+1}, \ldots, c_{n}\right), 1 \leq i \leq n$. Using condition (iii) and taking into consideration that, for each $i \in\{1, \ldots, n\}$, the section $S_{i}\left(c_{i}\right)$ is an open subset of $\prod_{j \neq i}^{n} K_{j}$, we obtain that $S_{i}\left(c_{i}\right) \times K_{i}$ is an open subset of $K$. Therefore, the set $F(c)=\bigcap_{i=1}^{n}\left(S_{i}\left(c_{i}\right) \times K_{i}\right)$ is open in $K$.

Suppose that $y \in K$. Write $c=\left(c_{1}, \ldots, c_{i-1}, c_{i}, c_{i+1}, \ldots, c_{n}\right)$ and note that since $c$ belongs to $F^{-1}(y)$ if and only if, for each $i \in\{1, \ldots, n\}$,

$$
c_{i} \in S_{i}\left(y_{1}, \ldots, y_{i-1}, y_{i+1}, \ldots, y_{n}\right)
$$

we have $F^{-1}(y)=\prod_{i=1}^{n} S_{i}\left(y_{1}, \ldots, y_{i-1}, y_{i+1}, \ldots, y_{n}\right)$. But, for each $i \in\{1, \ldots, n\}$, the sections $S_{i}\left(y_{1}, \ldots, y_{i-1}, y_{i+1}, \ldots, y_{n}\right)$ are nonempty convex subsets of $C_{i}$ by condition (ii), and thus, $F^{-1}(y)$ is a nonempty convex subset of $C$. We conclude that $F(C)=K$.

It follows from Theorem 2.2 that $u \in F(u)$ for some $u \in C$. This shows that, for each $i \in\{1, \ldots, n\}, u=\left(u_{1}, \ldots, u_{i-1}, u_{i}, u_{i+1}, \ldots, u_{n}\right) \in S_{i}$, that is, $u \in C \cap$ $\bigcap_{i=1}^{n} S_{i}$.

Example 4.2. Let $E=\mathbb{R}^{2}, n=2, K=K_{1} \times K_{2}$ where $K_{1}=K_{2}=[0 ; 1], C_{1}=C_{1,0} \cup$ $C_{1,1}$ where $C_{1,0}=[0 ; 1 / 3)$ and $C_{1,1}=(2 / 3 ; 1], C_{2}=[0 ; 1], C=C_{1} \times C_{2}, S=S_{1} \cup S_{2}$ where $S_{1}=K \cap\left\{\left(x_{1}, x_{2}\right): x_{2}>-3 x_{1}+2\right\}$, and $S_{2}=K \cap\left\{\left(x_{1}, x_{2}\right): x_{2}<-x_{1}+3 / 2\right\}$. Hence $C_{1}$ is a noncompact and nonconvex subset of $K_{1}$, the assumptions of Theorem 4.1 are satisfied and $C \cap \bigcap_{i=1}^{2} S_{i} \neq \varnothing$.

As an application of Theorem 4.1 we obtain the following theorem.

TheOREM 4.3. Let $E$ be a Hausdorff topological vector space over $\mathbb{R}$ and let $n \geq 2$. Let $C_{1}, \ldots, C_{n}$ be nonempty (not necessarily convex or compact) subsets of $E$, let $K_{1}, \ldots, K_{n}$ be convex compact subsets of $E$, and let $C=\prod_{j=1}^{n} C_{j}, K=\prod_{j=1}^{n} K_{j}$. Let $f_{1}, \ldots, f_{n}$ be real-valued maps defined on $K$, let $t_{1}, \ldots, t_{n}$ be real numbers, and let the following conditions hold:

(i) $C \subset K$;

(ii) for each $i, 1 \leq i \leq n$, and for each point $\left(y_{1}, \ldots, y_{i-1}, y_{i+1}, \ldots, y_{n}\right)$ of $\prod_{j \neq i}^{n} K_{j}$, the set $\left\{c_{i} \in C_{i}: f_{i}\left(y_{1}, \ldots, y_{i-1}, c_{i}, y_{i+1}, \ldots, y_{n}\right)>t_{i}\right\}$ is a nonempty convex subset of $C_{i}$; 
8 Fixed-point and coincidence theorems

(iii) for each $i, 1 \leq i \leq n$, and for each point $c_{i} \in C_{i}$, the set

$$
\left\{\left(y_{1}, \ldots, y_{i-1}, y_{i+1}, \ldots, y_{n}\right) \in \prod_{j \neq i} K_{j}: f_{i}\left(y_{1}, \ldots, y_{i-1}, c_{i}, y_{i+1}, \ldots, y_{n}\right)>t_{i}\right\}
$$

is an open subset of $\prod_{j \neq i}^{n} K_{j}$.

Then there is a point $u$ in $C$ such that $f_{i}(u)>t_{i}$ for each $i, 1 \leq i \leq n$.

Proof. Define the subsets $S_{i}$ of $K$ to be $S_{i}=\left\{y: y \in K, f_{i}(y)>t_{i}\right\}, i \in\{1, \ldots, n\}$. Clearly, (ii) is equivalent to the condition:

(ii') for each $i \in\{1, \ldots, n\}$ and for each point $\left(y_{1}, \ldots, y_{i-1}, y_{i+1}, \ldots, y_{n}\right)$ of $\prod_{j \neq i}^{n} K_{j}$, the section $S_{i}\left(y_{1}, \ldots, y_{i-1}, y_{i+1}, \ldots, y_{n}\right)$, formed by all points $c_{i} \in$ $C_{i}$ such that $\left(y_{1}, \ldots, y_{i-1}, c_{i}, y_{i+1}, \ldots, y_{n}\right) \in S_{i}$, is a nonempty convex subset of $K_{i}$,

and (iii) is equivalent to the condition:

(iii') for each $i \in\{1, \ldots, n\}$ and for each point $c_{i} \in C_{i}$, the section $S_{i}\left(c_{i}\right)$, formed by all points $\left(y_{1}, \ldots, y_{i-1}, y_{i+1}, \ldots, y_{n}\right)$ of $\prod_{j \neq i}^{n} K_{j}$ such that

$$
\left(y_{1}, \ldots, y_{i-1}, c_{i}, y_{i+1}, \ldots, y_{n}\right) \in S_{i}
$$

is an open subset of $\prod_{j \neq i}^{n} K_{j}$.

We can apply Theorem 4.1 to obtain $C \cap \bigcap_{i=1}^{n} S_{i} \neq \varnothing$. Hence, by the definition of $S_{i}$, the point $u$ from this intersection satisfies $f_{i}(u)>t_{i}$ for each $i \in$ $\{1, \ldots, n\}$.

\section{Coincidence theorems for set-valued maps and section theorems on not necessarily convex sets in topological vector spaces}

Using his infinite-dimensional version of the KKM theorem as a tool, Fan [16] established a geometrical "lemma" concerning convex and compact sets. Next, Browder [6] restated it in the more convenient form of a fixed-point theorem. A weaker form (with a relaxed compactness assumption) of this theorem was afterwards obtained by Fan [21]. Finally, Lassonde [33] extended these results. He gave a proof of the following interesting coincidence theorem:

Theorem 5.1. Let $X$ be a convex space (i.e., a convex set in a vector space with any topology that induces the Euclidean topology on the convex hulls of its finite subsets), $Y$ a topological space, and $F$ the map of $X$ into $2^{Y}$ for which the following conditions hold:

(i) for each $x \in X, F(x)$ is compactly open in $Y$;

(ii) for each $y \in Y, F^{-1}(y)=\{x \in X: y \in F(x)\}$ is nonempty and convex;

(iii) for some c-compact set $K \subset X$, the set $Y \backslash \bigcup_{x \in K} F(x)$ is compact. Then, for each single-valued continuous map $f$ of $X$ into $Y$, there exists an $x \in X$ such that $f(x) \in F(x)$. 
Our new coincidence theorem does not require convexity.

THEOREM 5.2. Let $C$ be a nonempty compact set in a Hausdorff topological vector space $E$ over $\mathbb{R}$ and let $f: C \rightarrow E$ be a continuous single-valued map on $C$ such that $f(C)$ is a convex set. Let $F: C \rightarrow 2^{f(C)}$ be a map such that $f(C)=F(C)$. Suppose that

(i) for each $y \in f(C)$, the set $F^{-1}(y)=\{c \in C: y \in F(c)\}$ is open in $C$;

(ii) for each $c \in C$, the set $\{y \in f(C): y \in F(c)\}$ is nonempty and convex.

Then there exists a point $u \in C$ such that $f(u) \in F(u)$.

Remark 5.3. If $f=I_{E}$ (the identity map) and $C$ is convex, then Theorem 5.2 becomes the Browder theorem [6, Theorem 1]. However, his method of proving this fact (based on the partition of unity) is absolutely different from ours.

Section theorems concerning convex compact sets in Hausdorff topological vector spaces, with various applications, are given by Fan $[18,20]$. In the proof of Theorem 5.2, we need the following two new auxiliary section theorems of Fan type.

Theorem 5.4. Let $C$ be a nonempty compact set (not necessarily convex) in a Hausdorff topological vector space E over $K$. Let $f: C \rightarrow E$ and $g: C \rightarrow E$ be continuous maps on $C$, and let $f(C)$ be convex. Let $K$ be a subset of $g(C) \times f(C)$ having the following properties:

(i) for each fixed $w \in f(C)$, the set $\{t \in C:(g(t), w) \in K\}$ is closed in $C$;

(ii) for each $t \in C,(g(t), f(t)) \in K$;

(iii) for any fixed $t \in C$, the set $\{w \in f(C):(g(t), w) \notin K\}$ is convex (or empty).

Then there exists a point $c \in C$ such that $\{g(c)\} \times f(C) \subset K$.

Proof. We use KKM set-valued maps. Define a map $H: f(C) \rightarrow 2^{E}$ as follows:

$$
H(w)=\{t \in C:(g(t), w) \in K\}, \quad w \in f(C) .
$$

Obviously, by (i), $H(w)$ is a compact subset of $C$ and thus $f(H(w))$ is a compact subset of $f(C)$ for each $w \in f(C)$. Let $\left\{w_{1}, \ldots, w_{m}\right\}$ be any finite and fixed subset of $f(C)$. We prove that $\operatorname{conv}\left\{w_{1}, \ldots, w_{m}\right\} \subset f\left(H\left(w_{1}\right)\right) \cup \cdots \cup f\left(H\left(w_{m}\right)\right)$. To this goal, we assume that $f(s) \in \operatorname{conv}\left\{w_{1}, \ldots, w_{m}\right\}$ but $f(s) \notin f\left(H\left(w_{1}\right)\right) \cup$ $\cdots \cup f\left(H\left(w_{m}\right)\right)$ for some $s \in C$. Then $s \notin H\left(w_{i}\right)$ for all $i=1, \ldots, m$, that is, $\left(g(s), w_{i}\right) \notin K$ for any $i=1, \ldots, m$. Therefore, by (iii), $w_{i}, i=1, \ldots, m$, are contained in a convex set $U=\{w \in f(C):(g(s), w) \notin K\}$. Consequently, $\operatorname{conv}\left\{w_{1}, \ldots, w_{m}\right\} \subset U$ and, in particular, $f(s) \in U$, that is, $(g(s), f(s)) \notin K$, which, by (ii), is impossible. We must have $f(s) \in f\left(H\left(w_{1}\right)\right) \cup \cdots \cup f\left(H\left(w_{m}\right)\right)$. By virtue of [16, Lemma 1, page 305], this yields $f(c) \in \bigcap\{f(H(w)): w \in f(C)\}$ for some $c \in C$ and we conclude that $\{g(c)\} \times f(C) \subset K$ for some $c \in C$. 
10 Fixed-point and coincidence theorems

Theorem 5.5. Let $C$ be a nonempty compact set (not necessarily convex) in a Hausdorff topological vector space $E$ over $\mathbb{K}$. Let $f: C \rightarrow E$ and $g: C \rightarrow E$ be continuous maps on $C$, and let $f(C)$ be convex. Let $B$ be a subset of $g(C) \times f(C)$ and suppose that

(i) for each fixed $y \in f(C)$, the set $\{c \in C:(g(c), y) \in B\}$ is open in $C$;

(ii) for any fixed $c \in C$, the set $\{y \in f(C):(g(c), y) \in B\}$ is nonempty and convex.

Then there exists a point $u \in C$ such that $(g(u), f(u)) \in B$.

Proof. Here, $B$ denotes a complement of the set $K$ in $g(C) \times f(C)$ where $K$ is defined in Theorem 5.4

Proof of Theorem 5.2. We define a set $B=\{(c, y) \in C \times f(C): y \in F(c)\}$ and apply Theorem 5.5 for $g=I_{E}$.

\section{Coincidences for upper semicontinuous set-valued maps on not necessarily convex sets in locally convex spaces}

Let $F: C \rightarrow 2^{E}$ and $G: C \rightarrow 2^{E}$ and let $\Phi: G(c) \times F(c) \rightarrow E$ for each $c \in C$. We say that maps $F$ and $G$ have a $\Phi$-coincidence if there exist $c \in C$ and $(u, v) \in$ $G(c) \times F(c)$ such that $\Phi(u, v)=0$; this point $c$ is called a $\Phi$-coincidence point for $F$ and $G$. In particular, a $\Phi$-coincidence point is a coincidence point if $\Phi$ is of the form $\Phi(u, v)=u-v$ for $(u, v) \in G(c) \times F(c)$, and $c \in C$.

We use these notations in the following theorem.

Theorem 6.1. Let $C$ be a nonempty compact (not necessarily convex) set in a locally convex Hausdorff topological vector space $E$ over $\mathbb{K}$. Let $\Gamma$ be the set of all continuous seminorms p on E. Let $F: C \rightarrow 2^{E}$ and $G: C \rightarrow 2^{E}$ be upper semicontinuous maps such that $F(c)$ and $G(c)$ are compact subsets of $E$ for each $c \in C$ and let, for each $c \in C$, the map $\Phi: G(c) \times F(c) \rightarrow E$ be continuous on $G(c) \times F(c)$.

(a) Then either $F$ and $G$ have a $\Phi$-coincidence or there exist $p \in \Gamma$ and $\lambda>0$ such that $p(\Phi(u, v))>\lambda$ for all $c \in C$ and all $(u, v) \in G(c) \times F(c)$.

(b) Then either $F$ and $G$ have $a \Phi$-coincidence or there exists $p \in \Gamma$ and, for any $c \in C$ and any $u \in G(c)$, there exists $v \in F(c)$ such that

$$
0<p(\Phi(u, v))=\operatorname{Min}\{p(\Phi(u, w)): w \in F(c)\}
$$

Proof. (a) If $F$ and $G$ do not have a $\Phi$-coincidence in $C$, then, for all $c \in C$, the set $\Phi(G(c) \times F(c))$ is compact and $0 \notin \Phi(G(c) \times F(c))$.

First, observe that

(i) for each $c \in C$, there exist $p_{c} \in \Gamma$ and $\lambda_{c}>0$ such that

$$
p_{c}(\Phi(u, v))>2 \lambda_{c} \quad \forall(u, v) \in G(c) \times F(c) .
$$


Indeed, for an arbitrary and fixed $w \in \Phi(G(c) \times F(c))$, there exists $p_{w} \in \Gamma$ such that $p_{w}(w) \neq 0$ and, by the continuity of $p_{w}$, there exist a neighbourhood $M_{w}$ of $w$ and $\mu_{w}>0$ such that $\mu_{w}=\operatorname{Inf}\left\{p_{w}(t): t \in M_{w}\right\}$. Since the family $\left\{M_{w}\right.$ : $w \in \Phi(G(c) \times F(c))\}$ is an open cover of a compact set of $\Phi(G(c) \times F(c))$, there exists a finite subset $\left\{w_{1}, \ldots, w_{m}\right\}$ of $\Phi(G(c) \times F(c))$ such that the family $\left\{M_{w_{i}}\right.$ : $i=1,2, \ldots, m\}$ covers $\Phi(G(c) \times F(c))$ and we may assume that

$$
p_{c}=\operatorname{Max}\left\{p_{w_{i}}: i=1, \ldots, m\right\}, \quad \lambda_{c}=\left(\frac{1}{4}\right) \operatorname{Min}\left\{\mu_{w_{i}}: i=1, \ldots, m\right\} .
$$

Now we prove that

(ii) for each $c \in C$, there exist $p_{c} \in \Gamma, \lambda_{c}>0$, and a neighbourhood $W_{c}$ of $c$, such that

$$
p_{c}(\Phi(u, v))>\lambda_{c} \quad \forall x \in W_{c} \cap C,(u, v) \in G(x) \times F(x) .
$$

Indeed, let $c \in C$ be arbitrary and fixed and we define open sets $A_{c}$ and $B_{c}$ as follows:

$$
A_{c} \times B_{c}=\left\{(u, v): p_{c}(\Phi(u, v))>\lambda_{c}\right\}
$$

where $p_{c}$ and $\lambda_{c}$ are as in (i). Since $F(c) \subset A_{c}, G(c) \subset B_{c}, F$, and $G$ are upper semicontinuous, there exist neighbourhoods $U_{c}$ and $V_{c}$ of $c$, such that $F(x) \subset A_{c}$ for $x \in U_{c} \cap C$, and $G(y) \subset B_{c}$ for $y \in V_{c} \cap C$. Consequently, we may assume that $W_{c}=U_{c} \cap V_{c}$.

Finally, for each $c \in C$, let $p_{c}, \lambda_{c}$, and $W_{c}$ be as in (ii). Since the family $\left\{W_{c}\right.$ : $c \in C\}$ is an open cover of a compact set of $C$, there exists a finite subset $\left\{c_{1}, \ldots\right.$, $\left.c_{n}\right\}$ of $C$ such that the family $\left\{W_{c_{i}}: i=1, \ldots, n\right\}$ covers $C$ and we may assume that

$$
p=\operatorname{Max}\left\{p_{c_{i}}: i=1, \ldots, n\right\}, \quad \lambda=\operatorname{Min}\left\{\lambda_{c_{i}}: i=1, \ldots, n\right\} .
$$

(b) If $F$ and $G$ do not have a $\Phi$-coincidence in $C$, let $p$ and $\lambda$ be as in (a) and let $c \in C$ be arbitrary and fixed. Observe that, for any $u \in G(c)$, the continuous map $p(\Phi(u, \cdot))$ attains its minimum on a compact set $F(c)$. Let $k: G(c) \times F(c) \rightarrow R$ be a map defined by the formula $k(u, v)=p(\Phi(u, v))-\operatorname{Min}\{p(\Phi(u, w)): w \in$ $F(c)\}$. Obviously, $k(u, v)>0$ for each $(u, v) \in G(c) \times F(c)$ and, for each $u \in G(c)$, there exists $v \in F(c)$ such that $k(u, v)=0$.

Example 6.2. Let $E=C, U=\{c \in E:|c|=1,|\operatorname{Arg}(c)| \leq \pi / 4\}, U_{1}=-U+2^{1 / 2}$, $V_{1}=\left\{w: w=t c, 0 \leq t \leq 1, c \in U_{1}\right\}$. Let $C=U_{1} \cup\left(-U_{1}\right)$ and let $F: C \rightarrow 2^{E}$, $G: C \rightarrow 2^{E}$ be defined by $F(c)=-V_{1}$ for $c \in U_{1}, F(c)=V_{1}$ for $c \in-U_{1}$, and $G=-F$. Then $F$ and $G$ satisfy the assumptions of Theorem 6.1(b) for $\Phi$ defined by $\Phi(u, v)=u-v,(u, v) \in G(c) \times F(c), c \in C$. The sets $C, F(C), G(C), F(c)$, and $G(c)$ are nonconvex for all $c \in C$. Moreover, $C \subset F(C), C \subset G(C)$, the sets $F(C)$ and $G(C)$ are not contained in $C$ and any $c \in C$ is a coincidence of $F$ and $G$. 


\section{Fixed-point and coincidence theorems}

\section{Coincidences and fixed points for continuous single-valued maps on not necessarily convex sets in locally convex spaces}

Two maps $f: C \rightarrow E$ and $g: C \rightarrow E$ have a $\Phi$-coincidence, where $\Phi: g(C) \times$ $f(C) \rightarrow E$, if $\Phi(g(c), f(c))=0$ for some $c \in C$; this point $c$ is called a $\Phi$-coincidence point for $f$ and $g$. In particular, a $\Phi$-coincidence point is a coincidence point if $\Phi$ is of the form $\Phi(u, v)=u-v$ for $(u, v) \in g(C) \times f(C)$. We say that $c \in C$ is a $\Phi$-fixed point for $f: C \rightarrow E$ where $\Phi: C \times f(C) \rightarrow E$, if $\Phi(c, f(c))=0$. In particular, a $\Phi$-fixed point is a fixed point if $\Phi$ is of the form $\Phi(c, v)=c-v$ for $(c, v) \in C \times f(C)$.

For maps defined on convex sets, there are many variations, generalizations, and applications (see, e.g., [1, 10, 13, 24, 25, 31, 32, 34, 36, 38, 41, 42, 43, 46]) of the well-known Fan minimax inequality [20], Hartman-Stampacchia variational inequality [26], and Iohvidov theorem [29].

In this section, we will give further applications of Theorem 5.4. In particular, we derive some minimax theorem (Theorem 7.1), Hartman-Stampacchia type variational inequalities (Theorem 7.2), and a theorem of Iohvidov type (Theorem 7.3(b)) for maps on not necessarily convex sets. One of them will be used later to prove new results concerning $\Phi$-coincidences and $\Phi$-fixed points (in particular, coincidences and fixed points) of continuous single-valued maps on not necessarily convex sets (Theorem 7.4).

A real map $\psi$, defined on a topological vector space $E$, is said to be lower semicontinuous (upper semicontinuous) on $E$ if, for each real number $\mu$, the set $\{x \in E: \psi(x)>\mu\}(\{x \in E: \psi(x)<\mu\})$ is open.

A real map, $\psi$ defined on a convex set $A$ of a vector space $E$, is said to be quasiconcave (quasi-convex) on $A$ if, for each real number $\mu$, the set $\{a \in A: \psi(a)>$ $\mu\}(\{a \in A: \psi(a)<\mu\})$ is convex.

As a consequence of Theorem 5.4, we obtain the following theorem.

Theorem 7.1. Let $C$ be a nonempty compact set (not necessarily convex) in a Hausdorff topological vector space $E$ over $\mathbb{K}$. Let $f: C \rightarrow E$ and $g: C \rightarrow E$ be continuous maps on $C$ and let $f(C)$ be convex.

(a) Let $\Psi: g(C) \times f(C) \rightarrow \mathbb{R}$ be a map such that (i) for each $v \in f(C), \Psi(\cdot, v)$ is a lower semicontinuous map on $g(C)$; (ii) for each $u \in g(C), \Psi(u, \cdot)$ is a quasiconcave map on $f(C)$. Then there exists $c \in C$ such that

$$
\operatorname{Sup}\{\Psi(g(c), f(t)): t \in C\} \leq \operatorname{Sup}\{\Psi(g(s), f(s)): s \in C\} .
$$

Let, additionally, (iii) $\Psi(g(s), f(s)) \leq 0$ for all $s \in C$. Then there exists $c \in C$ such that

$$
\Psi(g(c), f(t)) \leq 0 \quad \forall t \in C
$$

(b) Let $\Omega: g(C) \times f(C) \rightarrow \mathbb{R}$ be a map such that (iv) for each $v \in f(C)$, the map $\operatorname{Min}\{\Omega(\cdot, w): w \in f(C)\}-\Omega(\cdot, v)$ is lower semicontinuous on $g(C)$; (v) for 
each $u \in g(C), \Omega(u, \cdot)$ is a quasi-convex map on $f(C)$. Then there exists $c \in C$ such that

$$
\Omega(g(c), f(c)) \leq \Omega(g(c), w) \quad \forall w \in f(C) .
$$

Proof. (a) If $\mu=\operatorname{Sup}\{\Psi(g(s), f(s)): s \in C\}$, then the set $K$ defined by $K=\{(g(t)$, $f(s)) \in g(C) \times f(C): \Psi(g(t), f(s)) \leq \mu\}$ satisfies the assumptions of Theorem 5.4 and thus, there exists $c \in C$ such that $\{g(c)\} \times f(C) \subset K$, that is, $\Psi(g(c), v) \leq$ $\mu$ for all $v \in f(C)$. This yields the assertion.

(b) Let $\Psi(u, v)=\operatorname{Min}\{\Omega(u, w): w \in f(C)\}-\Omega(u, v)$. The map $\Psi$ satisfies conditions (i) and (ii) on $g(C) \times f(C)$. Moreover, for each $u \in g(C)$, there exists $v \in f(C)$ such that $\Psi(u, v)=0$ and, by the assertion of (a), we get

$$
\operatorname{Sup}\{\Psi(g(s), f(s)): s \in C\} \geq 0
$$

Thus, there exists $c \in C$ such that $\Psi(g(c), f(c)) \geq 0$.

If $E$ is a locally convex Hausdorff topological vector space over $\mathbb{K}$ and $E^{\prime}$ denotes the topological vector space of continuous linear functionals on $E$, let $\langle\lambda ; x\rangle$ denote the pairing between $\lambda$ in $E^{\prime}$ and $x$ in $E$.

Now, we show the following theorem.

TheOrem 7.2. Let $C$ be a nonempty compact set (not necessarily convex) in a locally convex Hausdorff topological vector space $E$ over $\mathbb{K}$. Let $f: C \rightarrow E$ and $g: C \rightarrow$ $E$ be continuous maps on $C$ and let $f(C)$ be convex. Let $\Phi: g(C) \times f(C) \rightarrow E$ be a continuous map such that

$$
\Phi\left(u, \mu_{1} v_{1}+\mu_{2} v_{2}\right)=\mu_{1} \Phi\left(u, v_{1}\right)+\mu_{2} \Phi\left(u, v_{2}\right)
$$

holds for all $u \in g(C), v_{1}, v_{2} \in f(C)$ and $\mu_{1} \geq 0, \mu_{2} \geq 0$ with $\mu_{1}+\mu_{2}=1$.

(a) If $\Gamma=\left\{p_{\alpha}: \alpha \in Z\right\}$ is the set of all continuous seminorms $p_{\alpha}$ on $E, \alpha \in Z$, $\left\{p_{\alpha_{1}}, p_{\alpha_{2}}, \ldots, p_{\alpha_{n}}\right\}$ is a finite subset of $\Gamma$ and $p_{\alpha}=p_{\alpha_{1}}+p_{\alpha_{2}}+\cdots+p_{\alpha_{n}}$, then there exists at least one $c \in C$ such that, for each $w \in f(C)$,

$$
p_{\alpha}[\Phi(g(c), f(c))] \leq p_{\alpha}[\Phi(g(c), w)]
$$

(b) Let $T: g(C) \rightarrow E^{\prime}$ be continuous. Then there exists $c \in C$ such that

$$
\begin{aligned}
\operatorname{Inf}\{\operatorname{Re}\langle(T \circ g)(c) ; \Phi(g(c), f(t))\rangle: t \in C\} \\
\quad \geq \operatorname{Inf}\{\operatorname{Re}\langle(T \circ g)(s) ; \Phi(g(s), f(s))\rangle: s \in C\} .
\end{aligned}
$$

Let, additionally, $\Phi(g(s), f(s))=0$ for all $s \in C$. Then there exists $c \in C$ such that

$$
\operatorname{Re}\langle(T \circ g)(c) ; \Phi(g(c), f(t))\rangle \geq 0 \quad \forall t \in C .
$$


14 Fixed-point and coincidence theorems

(c) Let $h: g(C) \rightarrow E$ and $L:\left[\left(I_{E}-h\right) \circ g\right](C) \rightarrow E^{\prime}$ be continuous. Then there exists $c \in C$ such that

$$
\begin{aligned}
\operatorname{Inf}\{\operatorname{Re}\langle L[g(c)-h(g(c))] ; \Phi(g(c), f(t))\rangle: t \in C\} \\
\geq \operatorname{Inf}\{\operatorname{Re}\langle L[g(s)-h(g(s))] ; \Phi(g(s), f(s))\rangle: s \in C\} .
\end{aligned}
$$

Let, additionally, $\Phi(g(s), f(s))=0$ for all $s \in C$. Then there exists $c \in C$ such that

$$
\operatorname{Inf}\{\operatorname{Re}\langle L[g(c)-h(g(c))] ; \Phi(g(c), f(t))\rangle: t \in C\} \geq 0 \quad \forall t \in C .
$$

Proof. (a) We denote $\Omega=p_{\alpha} \circ \Phi$ and use Theorem 7.1(b).

(b) Let $\Psi: g(C) \times f(C) \rightarrow \mathbb{R}$ be a map of the form

$$
\Psi(u, v)=-\operatorname{Re}\langle T(u) ; \Phi(u, v)\rangle .
$$

Then $\Psi$ satisfies the conditions of (iv) and (v) and, consequently, there exists $c \in C$ such that

$$
\begin{aligned}
\operatorname{Inf}\{ & \operatorname{Re}\langle(T \circ g)(c) ; \Phi(g(c), f(t))\rangle: t \in C\} \\
& \geq \operatorname{Inf}\{\operatorname{Re}\langle(T \circ g)(s) ; \Phi(g(s), f(s))\rangle: s \in C\} .
\end{aligned}
$$

(c) We use (b) for $T=L \circ\left(I_{E}-h\right)$.

Our new coincidence theorem does not require convexity.

Theorem 7.3. Let $C$ be a nonempty compact set (not necessarily convex) in a locally convex Hausdorff topological vector space $E$ over $\mathbb{K}$. Let $\Gamma$ be the set of all continuous seminorms $p$ on $E$. If $f: C \rightarrow E$ and $g: C \rightarrow E$ are continuous on $C$, if $f(C)$ is convex, and if $\Phi: g(C) \times f(C) \rightarrow E$ is a continuous map such that

$$
\Phi\left(u, \mu_{1} v_{1}+\mu_{2} v_{2}\right)=\mu_{1} \Phi\left(u, v_{1}\right)+\mu_{2} \Phi\left(u, v_{2}\right)
$$

holds for all $u \in g(C), v_{1}, v_{2} \in f(C)$ and $\mu_{1} \geq 0, \mu_{2} \geq 0$ with $\mu_{1}+\mu_{2}=1$, then

(a) either $f$ and $g$ have a $\Phi$-coincidence in $C$ or there exist $c \in C$ and $p \in \Gamma$, such that

$$
0<p[\Phi(g(c), f(c))]=\operatorname{Min}\{p[\Phi(g(c), w)]: w \in f(C)\}
$$

(b) if, for each $u \in g(C)$, there is some $v \in f(C)$ with $\Phi(u, v)=0$, then $f$ and $g$ have a $\Phi$-coincidence in $C$. 
Proof. (a) Denote $A_{\alpha}=\left\{t \in C: p_{\alpha}[\Phi(g(t), f(t))]=0\right\}, \alpha \in Z$. Obviously, $A_{\alpha}$ is a closed subset of $C$ and thus compact, $\alpha \in Z$. Assume that the second assertion does not hold, that is, for each point $t \in C$ and for any $p_{\alpha} \in \Gamma$ such that $p_{\alpha}[\Phi(g(t), f(t))]>0$, there exists a point $w \in f(C)$ with $p_{\alpha}[\Phi(g(t), w)]<$ $p_{\alpha}[\Phi(g(t), f(t))]$.

We then prove that the above yields the first assertion, that is, that

$$
\bigcap\left\{A_{\alpha}: \alpha \in Z\right\} \neq \varnothing
$$

To this aim, observe that if $\left\{p_{\alpha_{1}}, p_{\alpha_{2}}, \ldots, p_{\alpha_{n}}\right\}$ is a finite subset of $\Gamma$ and $p_{\alpha}=$ $p_{\alpha_{1}}+p_{\alpha_{2}}+\cdots+p_{\alpha_{n}}$, then, by (7.6), there exists at least one $c \in C$ such that, for all $t \in f(C)$, we have $p_{\alpha}[\Phi(g(c), f(c))] \leq p_{\alpha}[\Phi(g(c), t)]$. But, by the above assumption, if $p_{\alpha}[\Phi(g(c), f(c))]>0$, then, for some $w \in f(C)$, we obtain $0<$ $p_{\alpha}[\Phi(g(c), f(c))] \leq p_{\alpha}[\Phi(g(c), w)]<p_{\alpha}[\Phi(g(c), f(c))]$, which is impossible and thus, $p_{\alpha}[\Phi(g(c), f(c))]=0$. Consequently, $c \in A_{\alpha_{1}} \cap A_{\alpha_{2}} \cap \cdots \cap A_{\alpha_{n}}$. This yields $\bigcap\left\{A_{\alpha}: \alpha \in Z\right\} \neq \varnothing$.

Part (b) is a simple consequence of (a).

By using various maps $\Phi, f$, and $g$, a number of variations of Theorem 7.3 can be obtained, of which the following is typical.

Theorem 7.4. Let $C$ be a nonempty compact set (not necessarily convex) in a locally convex Hausdorff topological vector space $E$ over $\mathbb{K}$. Let $\Gamma$ be the set of all continuous seminorms $p$ on $E$.

(i) If $f: C \rightarrow E$ and $g: C \rightarrow E$ are continuous on $C$ and if $f(C)$ is convex, then we have (a) either $f$ and $g$ have a coincidence or there exist $c \in C$ and $p \in \Gamma$ such that $0<p[g(c)-f(c)]=\operatorname{Min}\{p[g(c)-w]: w \in f(C)\}$; (b) if, for each $c \in C$, there exists $\lambda \in \mathbb{K}$ such that $|\lambda|<1$ and $\lambda f(c)+(1-\lambda) g(c) \in f(C)$, then $f$ and $g$ have a coincidence.

(ii) If $f: C \rightarrow E$ is continuous on $C$, if $f(C)$ is convex, and if $\Phi: C \times f(C) \rightarrow E$ is a continuous map such that $\Phi\left(c, \mu_{1} v_{1}+\mu_{2} v_{2}\right)=\mu_{1} \Phi\left(c, v_{1}\right)+\mu_{2} \Phi\left(c, v_{2}\right)$ holds for all $c \in C, v_{1}, v_{2} \in f(C)$ and $\mu_{1} \geq 0, \mu_{2} \geq 0$ with $\mu_{1}+\mu_{2}=1$, then (c) either $f$ has $a \Phi$-fixed point in $C$ or there exist $c \in C$ and $p \in \Gamma$ such that $0<p[\Phi(c, f(c))]=$ $\operatorname{Min}\{p[\Phi(c, w)]: w \in f(C)\} ;(\mathrm{d})$ if, for each $c \in C$, there is some $v \in f(C)$ with $\Phi(c, v)=0$, then $f$ has a $\Phi$-fixed point in $C$.

(iii) If $f: C \rightarrow E$ is continuous on $C$ and if $f(C)$ is convex, then we have (e) either $f$ has a fixed point in $C$ or there exist $c \in C$ and $p \in \Gamma$, such that $0<p[c-$ $f(c)]=\operatorname{Min}\{p(c-w): w \in f(C)\} ;$ (f) if, for each $c \in C$, there exists $\lambda \in \mathbb{K}$ such that $|\lambda|<1$ and $\lambda f(c)+(1-\lambda) c \in f(C)$, then $f$ has a fixed point in $C$.

Proof. We prove only (b). Assume that the assertion does not hold, that is, $g(c) \neq$ $f(c)$ for any $c \in C$. By (a), there exist a point $c \in C$ and some $p \in \Gamma$, such that

$$
0<p[g(c)-f(c)]=\operatorname{Min}\{p[g(c)-w]: w \in f(C)\}
$$


Obviously, by the assumption, there is a number $\lambda \in \mathbb{K}$ such that $|\lambda|<1$ and $w=\lambda f(c)+(1-\lambda) g(c) \in f(C)$. But then $w-g(c)=\lambda[f(c)-g(c)]$ and, by (7.16), we get $0<p[g(c)-f(c)] \leq p[g(c)-w]=|\lambda| p[g(c)-f(c)]$ which is impossible because $|\lambda|<1$.

Example 7.5. Let $E=\mathbb{C}$,

$$
\begin{aligned}
C= & \left\{c \in E:|\operatorname{Arg}(c)-\pi| \leq \frac{\pi}{4}, \frac{1}{2} \leq|c| \leq 1\right\} \\
& \cup\left\{c \in E:|\operatorname{Arg}(c)| \leq \frac{\pi}{4},|c| \leq 2\right\},
\end{aligned}
$$

$g(c)=c$ and $f(c)=-(\bar{c})^{2}+a, c \in C$, where $a=2^{1 / 2}+14^{1 / 2}$. Then $C$ is compact but nonconvex, $f$ is not injective on $C, f(C)=\{c \in E:|c-a| \leq 4$, $\pi / 2 \leq \operatorname{Arg}(c-a) \leq 3 \pi / 2\}, f$ is neither expansive nor inner and $\operatorname{Fix}(f)=$ $\left\{\left[-1+(1+4 a)^{1 / 2}\right] / 2\right\}$. For all $c \in C$, the segment $[c, f(c)]$ contains at least two points of $f(C)$. Moreover, the set $\partial C$ contains an infinite subset $C_{0}$ such that, for all $c \in C_{0}$, the segment $[c, f(c)]$ contains only one point of $C$. Thus, the assumptions of Theorem 7.4(b) are satisfied, but the assumptions of [19, Theorems 1 and 3] are not.

\section{References}

[1] G. Allen, Variational inequalities, complementarity problems, and duality theorems, J. Math. Anal. Appl. 58 (1977), no. 1, 1-10.

[2] H. Ben-El-Mechaiekh and P. Deguire, General fixed point theorems for nonconvex setvalued maps, C. R. Acad. Sci. Paris Sér. I Math. 312 (1991), no. 6, 433-438.

[3] - Approachability and fixed points for nonconvex set-valued maps, J. Math. Anal. Appl. 170 (1992), no. 2, 477-500.

[4] C. Berge, Topological Spaces, Oliver and Boyd, Edinburgh and London, 1963.

[5] H. F. Bohnenblust and S. Karlin, On a theorem of Ville, Contributions to the Theory of Games (H. W. Kuhn and A. W. Tucker, eds.), Annals of Mathematics Studies, no. 24, Princeton University Press, New Jersey, 1950, pp. 155-160.

[6] F. E. Browder, The fixed point theory of multi-valued mappings in topological vector spaces, Math. Ann. 177 (1968), 283-301.

[7] Coincidence theorems, minimax theorems, and variational inequalities, Conference in Modern Analysis and Probability (New Haven, Conn., 1982), Contemp. Math., vol. 26, Amer. Math. Soc., Rhode Island, 1984, pp. 67-80.

[8] P. Cubiotti, Some remarks on fixed points of lower semicontinuous multifunctions, J. Math. Anal. Appl. 174 (1993), no. 2, 407-412.

[9] P. Deguire, Browder-Fan fixed point theorem and related results, Discuss. Math. Differential Incl. 15 (1995), no. 2, 149-162.

[10] P. Deguire, K.-K. Tan, and G. X.-Z. Yuan, The study of maximal elements, fixed points for $L_{S}$-majorized mappings and their applications to minimax and variational inequalities in product topological spaces, Nonlinear Anal. 37 (1999), no. 7, Ser. A: Theory Methods, 933-951.

[11] X. P. Ding and E. Tarafdar, Fixed point theorems and existence of equilibrium points of noncompact abstract economies, Nonlinear World 1 (1994), no. 3, 319-340. 
[12] Some coincidence theorems and applications, Bull. Austral. Math. Soc. 50 (1994), no. 1, 73-80.

[13] J. Dugundji and A. Granas, KKM maps and variational inequalities, Ann. Scuola Norm. Sup. Pisa Cl. Sci. (4) 5 (1978), no. 4, 679-682.

[14] _ Fixed Point Theory. I, Monografie Matematyczne, vol. 61, Państwowe Wydawnictwo Naukowe (PWN), Warsaw, 1982.

[15] K. Fan, Fixed-point and minimax theorems in locally convex topological linear spaces, Proc. Nat. Acad. Sci. U.S.A. 38 (1952), 121-126.

[16] A generalization of Tychonoff's fixed point theorem, Math. Ann. 142 (1961), 305-310.

[17] _ Sur un théorème minimax, C. R. Acad. Sci. Paris 259 (1964), 3925-3928 (French).

[18] Applications of a theorem concerning sets with convex sections, Math. Ann. 163 (1966), 189-203.

[19] Extensions of two fixed point theorems of F. E. Browder, Math. Z. 112 (1969), 234-240.

[20] _ A minimax inequality and applications, Inequalities, III (Proc. Third Sympos., Univ. California, Los Angeles, Calif., 1969; dedicated to the memory of Theodore S. Motzkin) (O. Shisha, ed.), Academic Press, New York, 1972, pp. $103-$ 113.

[21] - Fixed-point and related theorems for noncompact convex sets, Game Theory and Related Topics (Proc. Sem., Bonn and Hagen, 1978) (O. Moeschlin and D. Pallaschke, eds.), North-Holland, Amsterdam, 1979, pp. 151-156.

[22] - Some properties of convex sets related to fixed point theorems, Math. Ann. 266 (1984), no. 4, 519-537.

[23] I. L. Glicksberg, A further generalization of the Kakutani fixed point theorem, with application to Nash equilibrium points, Proc. Amer. Math. Soc. 3 (1952), 170174.

[24] C.-W. Ha, Minimax and fixed point theorems, Math. Ann. 248 (1980), no. 1, 73-77.

[25] - On a minimax inequality of Ky Fan, Proc. Amer. Math. Soc. 99 (1987), no. 4, 680-682.

[26] P. Hartman and G. Stampacchia, On some non-linear elliptic differential-functional equations, Acta Math. 115 (1966), 271-310.

[27] C. J. Himmelberg, Fixed points of compact multifunctions, J. Math. Anal. Appl. 38 (1972), 205-207.

[28] C. Horvath, Points fixes et coïncidences pour les applications multivoques sans convexité, C. R. Acad. Sci. Paris Sér. I Math. 296 (1983), no. 9, 403-406 (French).

[29] I. S. Iohvidov, On a lemma of Ky Fan generalizing the fixed-point principle of A. N. Tihonov, Dokl. Akad. Nauk SSSR 159 (1964), 501-504 (Russian), English translation: Soviet. Math. 5 (1964), 1523-1526.

[30] S. Kakutani, A generalization of Brouwer's fixed point theorem, Duke Math. J. 8 (1941), 457-459.

[31] G. Kassay and Z. Páles, A localized version of Ky Fan's minimax inequality, Nonlinear Anal. 35 (1999), no. 4, Ser. A: Theory Methods, 505-515.

[32] H.-M. Ko and K.-K. Tan, A coincidence theorem with applications to minimax inequalities and fixed point theorems, Tamkang J. Math. 17 (1986), no. 2, 37-45.

[33] M. Lassonde, On the use of KKM multifunctions in fixed point theory and related topics, J. Math. Anal. Appl. 97 (1983), no. 1, 151-201. 
[34] Z. F. Li and S. Y. Wang, A type of minimax inequality for vector-valued mappings, J. Math. Anal. Appl. 227 (1998), no. 1, 68-80.

[35] J. Nash, Non-cooperative games, Ann. of Math. (2) 54 (1951), 286-295.

[36] D. O'Regan, Coincidences for admissible and $\Phi^{*}$ maps and minimax inequalities, J. Math. Anal. Appl. 220 (1998), no. 1, 322-333.

[37] S. Park and J. S. Bae, On zeros and fixed points of multifunctions with noncompact convex domains, Comment. Math. Univ. Carolin. 34 (1993), no. 2, 257-264.

[38] S. Park, J. S. Bae, and H. K. Kang, Geometric properties, minimax inequalities, and fixed point theorems on convex spaces, Proc. Amer. Math. Soc. 121 (1994), no. 2, 429-439.

[39] S. Park and K. S. Jeong, A general coincidence theorem on contractible spaces, Proc. Amer. Math. Soc. 124 (1996), no. 10, 3203-3206.

[40] S. Park and H. Kim, Coincidence theorems for admissible multifunctions on generalized convex spaces, J. Math. Anal. Appl. 197 (1996), no. 1, 173-187.

[41] S. Reich, Fixed points in locally convex spaces, Math. Z. 125 (1972), 17-31.

[42] B. Ricceri and S. Simons (eds.), Minimax Theory and Applications, Nonconvex Optimization and its Applications, vol. 26, Kluwer Academic Publishers, Dordrecht, 1998.

[43] M.-H. Shih and K.-K. Tan, Minimax inequalities and applications, Differential Analysis in Infinite-Dimensional Spaces (Albany, N.Y., 1983), Contemp. Math., vol. 54, Amer. Math. Soc., Rhode Island, 1986, pp. 45-63.

[44] E. Tarafdar, An extension of Fan's fixed point theorem and equilibrium point of an abstract economy, Comment. Math. Univ. Carolin. 31 (1990), no. 4, 723-730.

[45] G. Q. Tian, Fixed point theorems for mappings with noncompact and nonconvex domains, J. Math. Anal. Appl. 158 (1991), no. 1, 161-167.

[46] X. Wu and F. Li, On Ky Fan's section theorem, J. Math. Anal. Appl. 227 (1998), no. 1, $112-121$.

[47] G. X.-Z. Yuan, The study of minimax inequalities and applications to economies and variational inequalities, Mem. Amer. Math. Soc. 132 (1998), no. 625, 140.

[48] G. X.-Z. Yuan, B. Smith, and S. Lou, Fixed point and coincidence theorems of set-valued mappings in topological vector spaces with some applications, Nonlinear Anal. 32 (1998), no. 2, 183-199.

[49] E. Zeidler, Nonlinear Functional Analysis and Its Applications. I. Fixed-Point Theorems, Springer-Verlag, New York, 1986.

[50] _ Nonlinear Functional Analysis and Its Applications. IV. Applications to Mathematical Physics, Springer-Verlag, New York, 1988.

Kazimierz Włodarczyk: Faculty of Mathematics, University of Łódź, Banacha 22, 90-238 Łódź, Poland

E-mail address: wlkzxa@imul.uni.lodz.pl

Dorota Klim: Faculty of Mathematics, University of Łódź, Banacha 22, 90-238 Łódź, Poland

E-mail address: klimdr@imul.uni.lodz.pl 


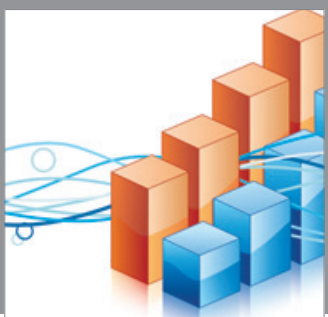

Advances in

Operations Research

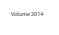

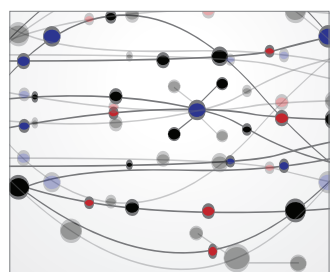

\section{The Scientific} World Journal
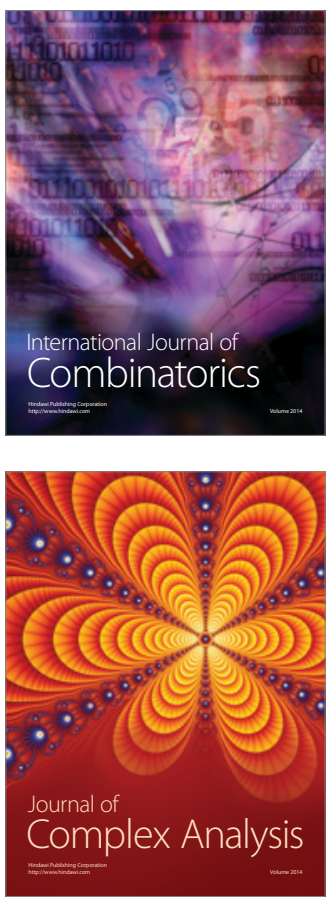

International Journal of

Mathematics and

Mathematical

Sciences
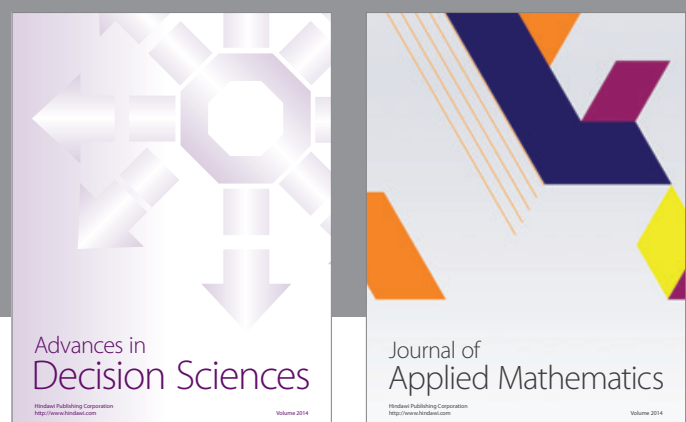

Journal of

Applied Mathematics
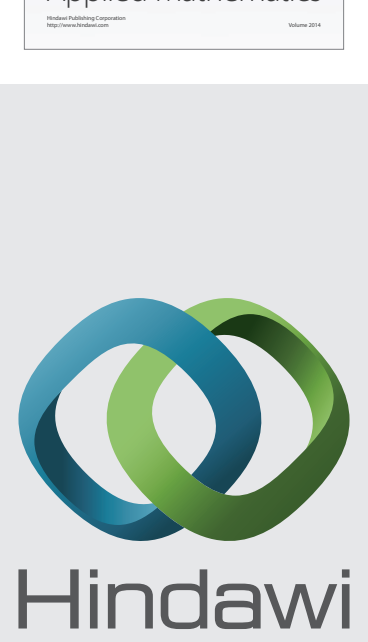

Submit your manuscripts at http://www.hindawi.com
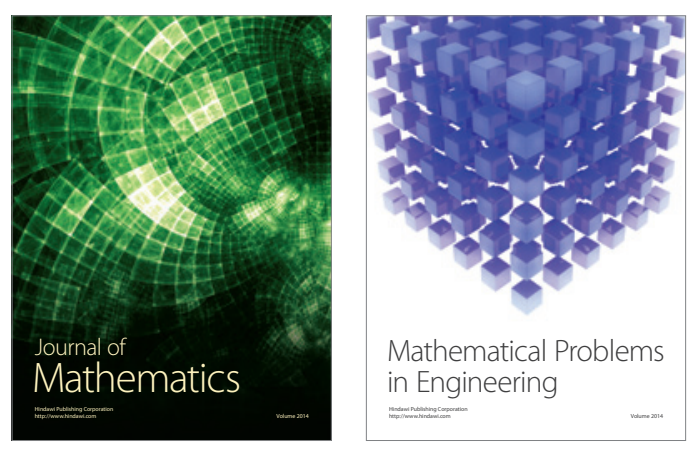

Mathematical Problems in Engineering
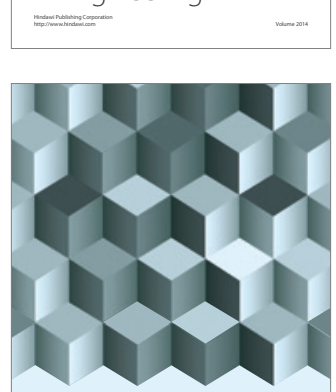

Journal of

Function Spaces
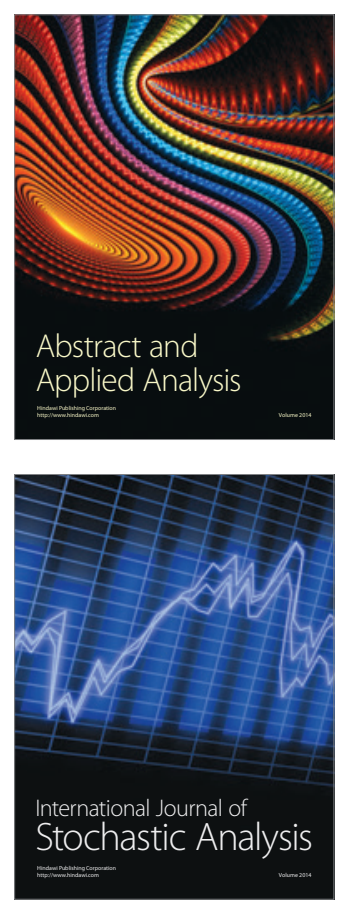

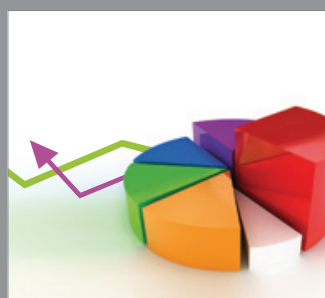

ournal of

Probability and Statistics

Promensencen
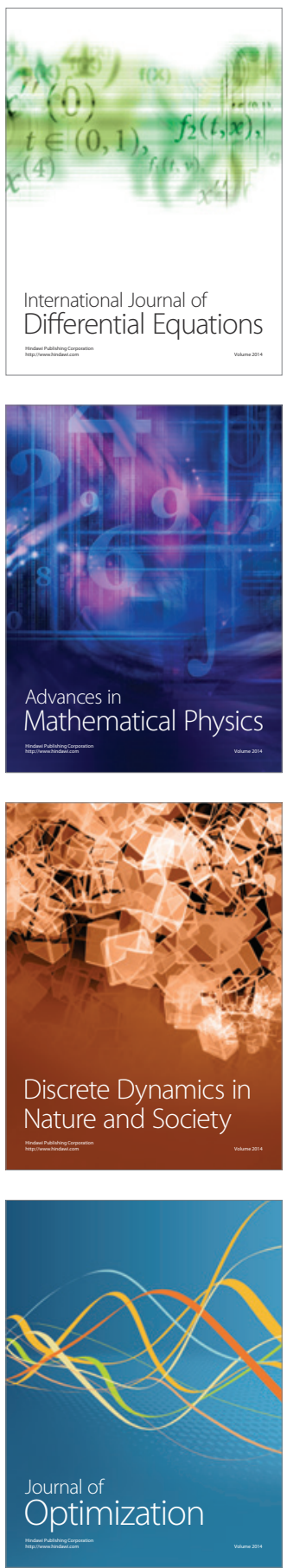